\title{
Ability of a neuro-ophthalmologist to estimate retinal nerve fiber layer thickness
}

This article was published in the following Dove Press journal:

Clinical Ophthalmology

10 September 2012

Number of times this article has been viewed

\author{
John H Pula' \\ Jorge C Kattah' \\ Hauping Wang' \\ John Marshall' \\ Eric R Eggenberger ${ }^{2}$ \\ 'University of Illinois College of \\ Medicine at Peoria, Illinois Neurologic \\ Institute, Peoria, IL, USA; ${ }^{2}$ Michigan \\ State University, East Lansing, \\ MI, USA
}

Background: Qualitative description of the optic disc has clinical value, but optical coherence tomography (OCT) has provided the ability to quantify retinal nerve fiber layer (RNFL) thickness.

Methods: We asked three neuro-ophthalmologists of at least 20 years' experience to estimate the average OCT RNFL thickness of 37 eyes based on fundus photos.

Results: The overall correlation coefficient for RNFL thickness estimation variance between two physicians and between physician and OCT was 0.53 . The likelihood that the RNFL thickness estimation between physicians, or between physician and OCT, was within $10 \mu \mathrm{m}$ of each other was $47 \%-62 \%$. All physicians had disparities in RNFL thickness estimation greater than $30 \mu \mathrm{m}$.

Conclusion: This study provides information on the ability of an experienced neuroophthalmologist to estimate the RNFL thickness based on fundus photos.

Keywords: optical coherence tomography, OCT, RNFL, retinal nerve fiber, estimation

\section{Introduction}

Optical coherence tomography (OCT) is an efficient method of quantifying retinal nerve fiber layer (RNFL) thickness. RNFL thickness can be used to measure atrophy after optic neuritis ${ }^{1,2}$ and as a marker for generalized central nervous system degeneration in multiple sclerosis. ${ }^{3-5}$ RNFL thickness in eyes with optic atrophy reflects both the remaining functional nerves and residua such as glial remnants or nonfunctioning axons. ${ }^{6}$ These anatomic measurements have a physiologic correlation, as average RNFL thickness decreases in proportion to visual field loss after optic neuritis, probably reflecting loss of functional axons. ${ }^{2}$ Thus, the objective measurements found with OCT are becoming more important in the setting of performing clinical trials. The range of RNFL thickness in nonedematous eyes is roughly $110 \mu \mathrm{m}$ at maximum, and at minimum, as in eyes with no light perception after longstanding optic neuropathy, average RNFL thickness is approximately $45 \mu \mathrm{m} .^{7}$

Our objective was to compare the ability of experienced neuro-ophthalmologists to estimate average RNFL thickness of eyes based on fundus photos showing the optic discs and peripapillary region and compare their result to the OCT measurement. We anticipated that agreement for physician estimates of RNFL thickness from fundus photos would not be superior to concordance measurements previously found for OCT, but we were particularly interested in the extent of correlation, as there has been no previous study quantifying this measurement.
Department of Neurology and Neuroophthalmology, University of Illinois College of Medicine at Peoria, Illinois Neurologic Institute, 530 NE Glen Oak Avenue, Peoria, IL 61637, USA

$\mathrm{Tel}+\mathrm{I} 3096552164$

Fax +I 3096552040

Email jpula I@uic.edu 


\section{Methods}

Institutional review board approval for this study was granted by a university internal review board. Data present in this manuscript were, in part, presented as an abstract for the 2012 North American Neuro-Ophthalmology Society Meeting in San Antonio, TX, as Poster 55.

Digital photographs and OCT were obtained from 37 eyes, which were selected based on gathering a range of RNFL thickness between 35 and $110 \mu \mathrm{m}$. We included eyes in which fundus photos and OCT were both performed on the same day. Fundus photos including optic disc and peripapillary region were all performed with a Visucam digital camera (Carl Zeiss, Thornwood, NY) using a standard color capture mode with a -2 correction flash and $30^{\circ}$ angle. OCT was acquired using a Carl Zeiss Stratus OCT Fast RNFL thickness profile to generate an average RNFL thickness. Only OCT RNFL measurements with a manufacturer's signal strength score of at least 7 with a properly centered scan circle were selected.

Photos of the optic discs were digitized and sent through electronic mail to three practising neuro-ophthalmologists with clinical experience of 21,26 , and 29 years, respectively, who were asked to estimate the OCT average RNFL thickness based only on the standard color fundus photos. The three estimating physicians were not the authors of this study, nor had they previously seen the fundus photos or subjects whose eyes were used. No clinical or paraclinical data were available for the physician to produce an estimation. Prior to data collection it was determined that an estimation of under $75 \mu \mathrm{m}$ would be used as a threshold of disc atrophy. If an OCT estimation was recorded as under $75 \mu \mathrm{m}$, it would be labeled as "atrophic." An OCT estimation over $75 \mu \mathrm{m}$ would be labeled as "not atrophic." This thickness threshold was based on a value of less than or equal to $75 \mu \mathrm{m}$, being the OCT thickness corresponding to approximately the fifthlowest percent of normal.

\section{Statistical analysis}

We used the Bland-Altman method to calculate the limits of agreement between the RNFL thickness estimation of each physician and the OCT RNFL thickness. To obtain a specific index to summarize the agreement degree, we used both Shrout and Fleiss intraclass correlation coefficient (ICC) and Lint's concordance correlation coefficient (CCC). ${ }^{8}$ Barnhart's overall CCC was used to calculate agreement of RNFL thickness estimation among the three physicians. Shrout and Fleiss ICC was used to calculate the ICC. ${ }^{9}$ A Levene test was used to check the homogeneity assumption for the three ICCs.

\section{Results}

Thirty-seven fundus photos (16 right eyes and 21 left eyes) from 26 subjects (17 female, nine male) were studied. Both eyes were used from nine subjects, and for the remaining 19 , one eye was used. The median patient age was 49 years (range 20-75 years). Table 1 reports the number of eyes for each OCT-determined RNFL thickness range as well as the primary diagnosis for each eye.

The agreement index compared with OCT based on the CCC for physician A was 0.53 (95\% confidence interval [CI]: 0.30-0.70). For physician B, agreement index was 0.43 (95\% CI: 0.14-0.65). For physician C, agreement index was 0.72 (95\% CI: 0.53-0.84). CCC and ICC data are represented in Table 2. Figure 1 displays CCCs for the three physicians. The overall $\mathrm{CCC}$ reflecting agreement among the three physicians was 0.53 with a $95 \%$ bootstrap CI of 0.29-0.69. Two thousand bootstrap samples created from the original data set with replacement were used to create the $95 \%$ CI.

OCT RNFL thickness ranged from $37.7 \mu \mathrm{m}$ to $104.6 \mu \mathrm{m}$. Bland-Altman analysis determined a limit of agreement for OCT and physician A of -32.57 to 42.96. Limit of agreement for OCT and physician B was -33.09 to 40.77 . Limit of agreement for OCT and physician C was -27.70 to 18.89. Figure 2 displays Bland-Altman plots for the three physicians.

Table I Eyes used for photo estimation and OCT analysis, including the number of eyes used within each RNFL thickness range, and the primary diagnosis for each eye

\begin{tabular}{|c|c|c|}
\hline $\begin{array}{l}\text { OCT RNFL thickness } \\
\text { range }(\mu \mathrm{m})\end{array}$ & $\begin{array}{l}\text { Number of } \\
\text { eyes }\end{array}$ & Primary diagnosis \\
\hline $30-40$ & 1 & MS and drusen \\
\hline $40-50$ & 1 & ON of unknown etiology \\
\hline \multirow[t]{3}{*}{$50-60$} & 10 & 3 drusen \\
\hline & & 5 nonarteritic ischemic $\mathrm{ON}$ \\
\hline & & $2 \mathrm{MS}$ \\
\hline \multirow[t]{5}{*}{$60-70$} & 11 & 3 drusen \\
\hline & & 2 NA-AION \\
\hline & & I MS \\
\hline & & I optic neuritis and MS \\
\hline & & $3 \mathrm{ON}$ of unknown etiology \\
\hline \multirow[t]{3}{*}{$70-80$} & 5 & 2 NA-AION \\
\hline & & I tobacco-alcohol amblyopia \\
\hline & & 2 diabetic papillitis \\
\hline \multirow[t]{2}{*}{$80-90$} & 3 & I fellow eye with NA-AION \\
\hline & & 2 headache \\
\hline \multirow[t]{2}{*}{$90-100$} & 5 & $3 \mathrm{MS}$ \\
\hline & & 2 headache \\
\hline $100-110$ & 1 & MS \\
\hline
\end{tabular}

Abbreviations: MS, multiple sclerosis; NA-AION, nonarteritic ischemic optic neuropathy; OCT, optical coherence tomography; ON, optic neuropathy; RNFL thickness, retinal nerve fiber layer thickness. 
Table 2 CCC and ICC values with $95 \%$ confidence intervals for each physician compared with OCT, and overall coefficient, which combines all three physicians

\begin{tabular}{lllll}
\hline & CCC & CCC 95\% CI & ICC & ICC 95\% CI \\
\hline Dr A and OCT & 0.53 & $0.30-0.70$ & 0.54 & $0.27-0.73$ \\
Dr B and OCT & 0.43 & $0.14-0.65$ & 0.43 & $0.14-0.66$ \\
Dr C and OCT & 0.72 & $0.53-0.84$ & 0.72 & $0.51-0.85$ \\
Overall coefficient & 0.53 & $0.29-0.69$ & 0.53 & $0.34-0.70$ \\
\hline
\end{tabular}

Notes: For both CCC and ICC, the closer a value to I.00, the higher the degree of correlation.

Abbreviations: $\mathrm{CCC}$, concordance correlation coefficient; $\mathrm{Cl}$, confidence interval; ICC, intraclass correlation coefficient; OCT, optical coherence tomography.

Between physicians, the likelihood was $57.7 \%$ for estimating an RNFL value within $10 \mu \mathrm{m}$ of each other, and $83.8 \%$ that the estimate would be within $20 \mu \mathrm{m}$ of each other. There was a $52.3 \%$ likelihood among all physicians for an estimation within $10 \mu \mathrm{m}$ of the OCT, and $82.0 \%$ for an estimation within $20 \mu \mathrm{m}$ between physician and OCT. Table 3 displays the range of variation among physicians and OCT. The chance of underestimating and overestimating the OCT result for all physicians was equal at 50\%.

Twenty-eight photos represented eyes with an OCT RNFL thickness of $75 \mu \mathrm{m}$ or less. Sixty-three of 84 (75\%) of the estimations based on these photos were rated as $75 \mu \mathrm{m}$ or less by the physicians. Forty-one of $51(80 \%)$ of the estimations based on photos of eyes with an OCT RNFL thickness of less than $65 \mu \mathrm{m}$ were rated as $75 \mu \mathrm{m}$ or less by the physicians. An OCT RNFL thickness measurement of $53 \mu \mathrm{m}$ was the threshold of thinning after which all physicians estimated the RNFL thickness as $75 \mu \mathrm{m}$ or less. Fifteen of 15 (100\%) of physicians rated these RNFL thicknesses as less than or equal to $75 \mu \mathrm{m}$. Eyes with OCT RNFL thickness measurements of greater than $53 \mu \mathrm{m}$ had at least one physician RNFL thickness estimation of $75 \mu \mathrm{m}$ or more.

Nine photos represented eyes with an OCT RNFL thickness of greater than $75 \mu \mathrm{m}$. Nineteen of $27(70 \%)$ estimations based on these photos were rated as greater than $75 \mu \mathrm{m}$ by the physicians.

\section{Discussion}

To our knowledge, there has been no prior study examining the ability of a neuro-ophthalmologist to estimate the average peripapillary RNFL thickness found on OCT, based on fundus photos. Nor has there been a study to compare this estimation

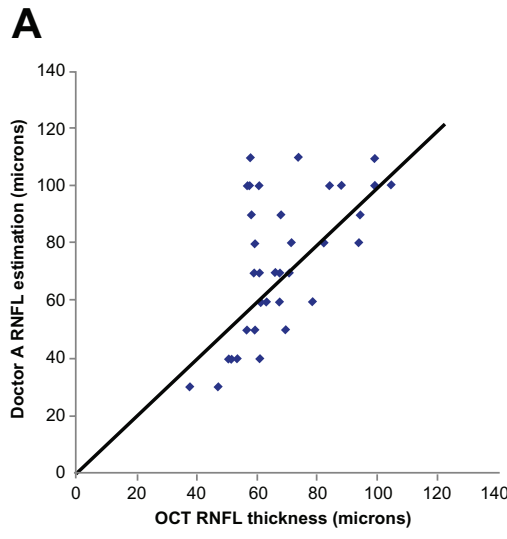

B

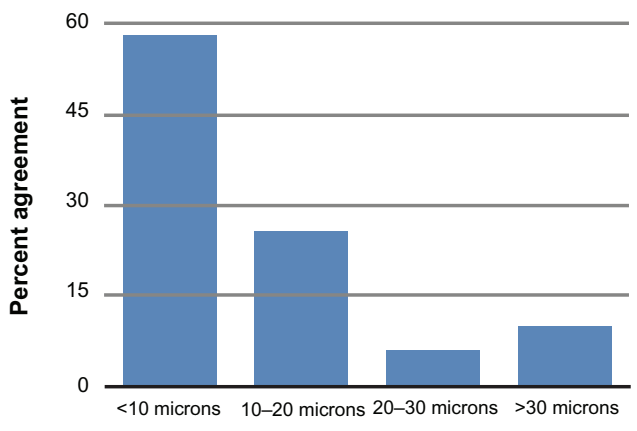

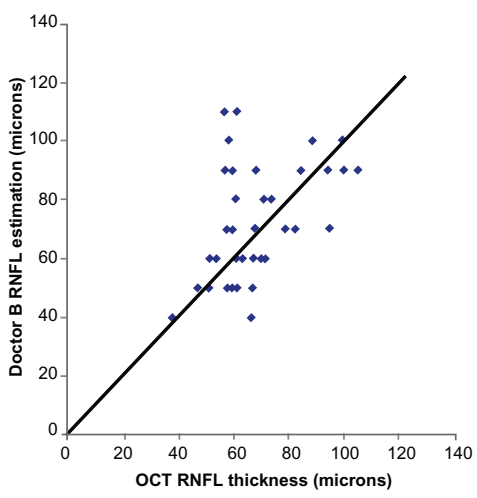

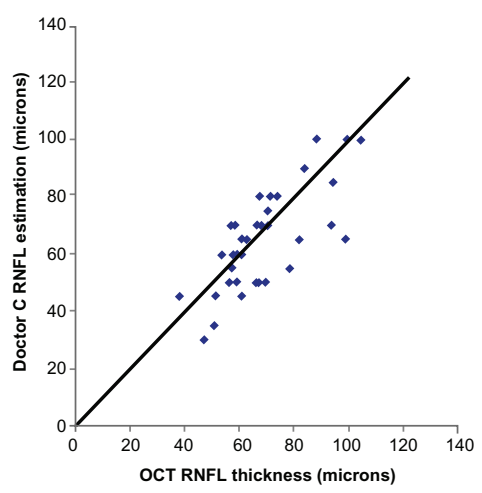

C

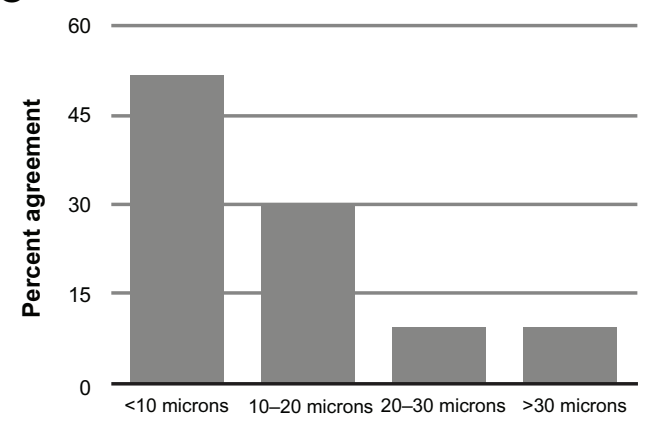

Figure I (A) Correlation graphs of retinal nerve fiber layer (RNFL) thickness estimations for physicians A, B, and C compared with optical coherence tomography (OCT). An exact reproducibility between physician and OCT results in a point plotted on the $45^{\circ}$ graph line. A physician overestimation compared with OCT plots above the line, and an underestimation plots under the line. The further away a point is from the $45^{\circ}$ line, the greater the difference between the RNFL estimation of the physician from the OCT. Below, percent of eyes (x-axis) that had RNFL thickness estimation agreement for each range in the $y$-axis between (B) two physicians and (C) physician and OCT. 

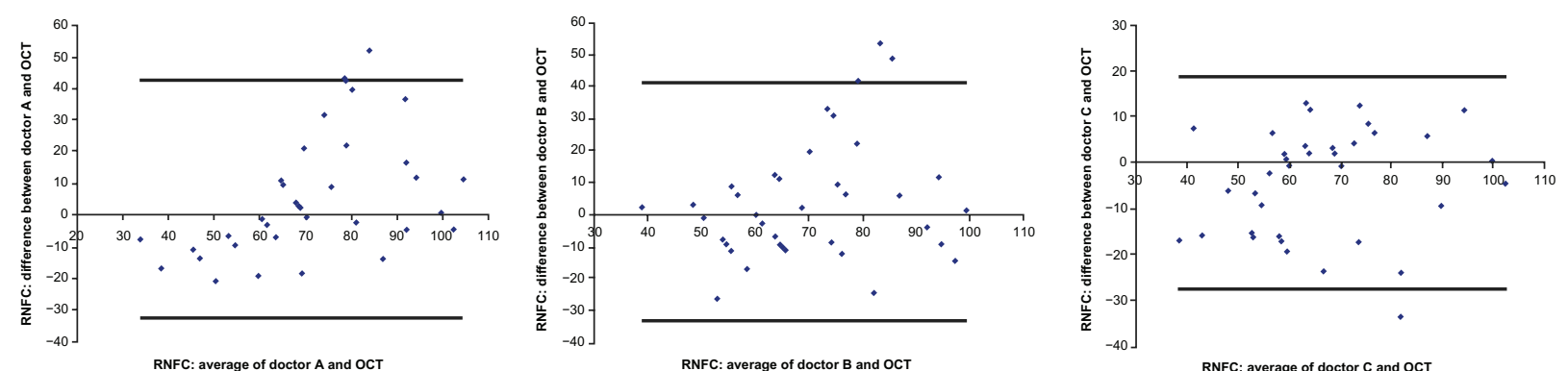

Figure 2 Bland-Altman graph representing limits of agreement for physicians A, B, and C compared with optical coherence tomography (OCT).

Notes: Average OCT for each comparison is plotted on the x-axis; difference between the physician and OCT score is on the $y$-axis, calculated as physician - OCT. Twice above and below standard deviation of the means is represented by the solid lines. The further away from zero a point plots on the $y$-axis, the greater the difference that point is between the rater and OCT.

Abbreviation: RNFC, retinal nerve fiber layer thickness composite.

with that of other neuro-ophthalmologists of similar clinical experience.

In a different study from ours, physicians detected optic nerve pallor with a sensitivity of $32 \%$ and specificity of $92 \%$ for eyes in which pallor was defined as having an average OCT RNFL thickness under the fifth percentile of normal. For eyes with an average RNFL thickness under the first percentile of normal, sensitivity improved slightly to $44 \%$, and specificity was $91 \%$. Estimators in this study ranged in experience from residents to attending neurology faculty, and direct ophthalmoscopes were used. ${ }^{10}$ Although that study serves as a real-world estimation for the likelihood of detecting optic atrophy, ours is a more specific examination of the ability to estimate the OCT RNFL thickness.

We did not ask our estimators to determine whether a disc was "atrophic" or not. Nor did we report to them the RNFL thickness that we would consider "atrophic." By defining optic atrophy in our study as an OCT value of less than or equal to $75 \mu \mathrm{m}$, a thickness corresponding to approximately the fifth-lowest percent of normal, our estimators had a sensitivity of $75 \%$ in estimating RNFL thickness under

Table 3 Range of RNFL thickness estimation difference between two physicians and between each individual physician and OCT

\begin{tabular}{lllll}
\hline & $\begin{array}{l}\text { Percent of } \\
\text { estimations } \\
\text { differing by } \\
\mathbf{0 - 1 0 \mu \mathbf { m }}\end{array}$ & $\mathbf{I I - 2 0} \boldsymbol{\mu m}$ & $\mathbf{2 I - 3 0 \mu m}$ & $>\mathbf{3 0} \boldsymbol{\mu m}$ \\
\hline Dr A and Dr B & $62 \%$ & $22 \%$ & $8 \%$ & $8 \%$ \\
Dr B and Dr C & $51 \%$ & $35 \%$ & $3 \%$ & $11 \%$ \\
Dr A and Dr C & $59 \%$ & $22 \%$ & $8 \%$ & $11 \%$ \\
Dr A and OCT & $47 \%$ & $31 \%$ & $8 \%$ & $14 \%$ \\
Dr B and OCT & $51 \%$ & $27 \%$ & $8 \%$ & $14 \%$ \\
Dr C and OCT & $59 \%$ & $32 \%$ & $5 \%$ & $3 \%$ \\
\hline Ab & & & & \\
\hline
\end{tabular}

Abbreviations: OCT, optical coherence tomography; RNFL thickness, retinal nerve fiber layer thickness.
$75 \mu \mathrm{m}$ when the OCT RNFL thickness was less than or equal to $75 \mu \mathrm{m}$. When the OCT RNFL thickness was $65 \mu \mathrm{m}$ or less, the sensitivity increased to $80 \%$ that the physician would estimate the RNFL thickness as $75 \mu \mathrm{m}$ or less. The RNFL thickness in which there was $100 \%$ sensitivity that our physicians would estimate the RNFL thickness at $75 \mu \mathrm{m}$ or less was $54 \mu \mathrm{m}$ on OCT.

Some clinical scales, such as the Kurtzke functional system scores, and the expanded disability scale for multiple sclerosis, use the presence or absence of optic atrophy as a factor of rating disability. Using the cut off that an OCT thickness of $75 \%$ normal represents atrophy, our results suggest that even in experienced raters, up to $25 \%$ of discs may be discordantly interpreted between two physicians. Complementing clinical scales with OCT may serve to minimize variability.

CCC and ICC measure reproducibility, where the degree of reproducibility improves as the agreement index approaches 1.0. Prior studies have evaluated the reproducibility of OCT between trials and between examiners. Cettomai et $\mathrm{al}^{11}$ reported an ICC of 0.89 for interrater reproducibility of OCT measurements with a $95 \mathrm{CI}$ for each eye ranging between 0.72 and 0.96 . This ICC result for OCT outperforms the overall reproducibility ICC of our three physicians of 0.56 (95\% CI: 0.38-0.72). CCC has some advantages over ICC for correlation analysis; thus, we also calculated CCC scores, which did not differ significantly from ICC. It should be noted that when variance of the sample exceeds that of the population, both CCC and ICC may overestimate agreement. We did not expect physician coefficients to be superior to OCT, but results showed that physician estimations were often very close to OCT, although at times were widely discordant.

Despite many advantages, there remain several shortcomings to OCT for evaluation of the optic disc. Tilted discs or healthy, normal-appearing discs with 
anomalous peripapillary choroidal degeneration may show thinning of the RNFL on stratus OCT. ${ }^{12}$ Spectral-domain OCT may be superior in this setting. Nevertheless, without a corresponding clinical exam, over-reliance on OCT results could lead to erroneous extrapolations of optic nerve health. Also, disc edema is presently suboptimally measured by OCT due to limits of recording, and the Frisen scale remains the standard for grading papilledema. Several studies have compared Frisen grade with OCT-measured edema, with mixed results. ${ }^{13}$ To avoid this issue, we did not use edematous discs in our investigation.

Limitations of our study include the use of fundus photos as a surrogate for real-life viewing of the fundus to estimate the OCT average RNFL thickness. Fundus photos may be inferior to binocular viewing of the disc, but practicality in study design mandated the use of photos. Second, we sampled only three neuro-ophthalmologists as estimators. Clearly, more estimators would have provided a greater sampling of data, but by using a relatively large number of eye estimations per physician, we were able to obtain significantly meaningful data. Third, we estimated average OCT RNFL thickness which rarely represents evenly distributed atrophy. Usually there is sectoral (focal) atrophy with other areas of relatively normal RNFL thickness. We did not ask for estimations of sectoral thickness in our study, but this is a possible point for future investigation.

\section{Conclusion}

Although neuro-ophthalmologists are quite capable of estimating average OCT RNFL thickness based solely on fundus photos, responses can vary widely, and up to $20 \%$ of the time, the responses will disagree with each other or with OCT by at least $20 \mu \mathrm{m}$. We underscore that OCT is a valuable objective tool that is meant to supplement, not replace, the clinical exam.

\section{Disclosure}

There is no associated funding regarding this work. The authors declare no conflicts of interest.

\section{References}

1. Costello F, Coupland S, Hodge W, et al. Quantifying axonal loss after optic neuritis with optical coherence tomography. Ann Neurol. 2006;59: 963-969.

2. Costello F, Hodge W, Pan YI, Eggenberger E, Coupland S, Kardon RH. Tracking retinal nerve fiber layer loss after optic neuritis: a prospective study using optical coherence tomography. Mult Scler. 2008;14: 893-905.

3. Albrecht P, Fröhlich R, Hartung H-P, Kieseier BC, Methner A. Optical coherence tomography measures axonal loss in multiple sclerosis independently of optic neuritis. $J$ Neurol. 2007;254:1595-1596.

4. Frohman EM, Fujimoto JG, Frohman TC, Calabresi PA, Cutter G, Balcer LJ. Optical coherence tomography: a window into the mechanisms of multiple sclerosis. Nat Clin Pract Neurol. 2008;4(12):664-675.

5. Sergott RC, Frohman E, Glanzman R, AL-Sabbagh A. The role of optical coherence tomography in multiple sclerosis: expert panel consensus. J Neurol Sci. 2007;263:3-14.

6. Cursiefen C, Holbach LM, Schlotzer-Schrehardt U, et al. Persisting retinal ganglion cell axons in blind atrophic human eyes. Graefes Arch Clin Exp Ophthalmol. 2001;239:158-164.

7. Chan CK, Miller NR. Peripapillary nerve fiber layer thickness measured by optical coherence tomography in patients with no light perception from long-standing nonglaucomatous optic neuropathies. J Neuroophthalmol. 2007;27(3):176-179.

8. Crawford SB, Kosinski AS, Lin HM, Williamson JM, Barnhart HX. Computer programs for the concordance correlation coefficient. Comput Methods Programs Biomed. 2007;88(1):62-74.

9. Shrout PE, Fleiss JL. Intraclass correlations: uses in assessing rater reliability. Psychol Bull. 1979;86:420-428.

10. Cettomai D, Hiremath G, Ratchford J, Venkatesan A, Greenberg BM, McGready J, et al. Associations between retinal nerve fiber layer abnormalities and optic nerve examination. Neurology. 2010;75(15): 1318-1325.

11. Cettomai D, Pulicken M, Gordon-Lipkin E, et al. Reproducibility of optical coherence tomography in multiple sclerosis. Arch Neurol. 2008;65(9):1218-1222.

12. Pula JH, Marshall J, Wang H, Kattah JC. Optical coherence tomography and visual field patterns in eyes with anomalous optic discs and acquired optic neuropathy. Neuro-ophthalmology. 2012;36(1):9-13.

13. Scott CJ, Kardon RH, Lee AG, Frisen L, Wall M. Diagnosis and grading of papilledema in patients with raised intracranial pressure using optical coherence tomography vs clinical expert assessment using a clinical staging scale. Arch Ophthalmol. 2010;128(6):705-711.
Clinical Ophthalmology

\section{Publish your work in this journal}

Clinical Ophthalmology is an international, peer-reviewed journal covering all subspecialties within ophthalmology. Key topics include: Optometry; Visual science; Pharmacology and drug therapy in eye diseases; Basic Sciences; Primary and Secondary eye care; Patient Safety and Quality of Care Improvements. This journal is indexed on

Submit your manuscript here: http://www.dovepress.com/clinical-ophthalmology-journal

\section{Dovepress}

PubMed Central and CAS, and is the official journal of The Society of Clinical Ophthalmology (SCO). The manuscript management system is completely online and includes a very quick and fair peer-review system, which is all easy to use. Visit http://www.dovepress.com/ testimonials.php to read real quotes from published authors. 